\title{
A Global Curvature Pinching Result of the First Eigenvalue of the Laplacian on Riemannian Manifolds
}

\author{
Peihe Wang ${ }^{1}$ and Ying $\mathrm{Li}^{2}$ \\ ${ }^{1}$ School of Mathematical Sciences, Qufu Normal University, Shandong, Qufu 273165, China \\ ${ }^{2}$ College of Science, University of Shanghai for Science and Technology, Shanghai 200093, China \\ Correspondence should be addressed to Peihe Wang; peihewang@hotmail.com
}

Received 8 December 2012; Revised 19 February 2013; Accepted 9 March 2013

Academic Editor: Wenming Zou

Copyright (c) 2013 P. Wang and Y. Li. This is an open access article distributed under the Creative Commons Attribution License, which permits unrestricted use, distribution, and reproduction in any medium, provided the original work is properly cited. The paper starts with a discussion involving the Sobolev constant on geodesic balls and then follows with a derivation of a lower
bound for the first eigenvalue of the Laplacian on manifolds with small negative curvature. The derivation involves Moser iteration.

\section{Introduction}

The Laplacian is one of the most important operator on Riemannian manifolds, and the study of its first eigenvalue is also an interesting subject in the field of geometric analysis. In general, people would like to estimate the first eigenvalue of Laplacian in terms of geometric quantities of the manifolds such as curvature, volume, diameter, and injectivity radius. In this sense, the first interesting result is that of Lichnerowicz and Obata, which proved the following result in [1]: let $M^{n}$ be an $n$-dimensional compact Riemannian manifold without boundary with $\operatorname{Ric}(M) \geq(n-1)$, then the first eigenvalue of Laplacian on $M^{n}$ will satisfy that $\lambda_{1}(M) \geq n$, and the inequality becomes equality if and only if $M^{n} \cong S^{n}$.

The above result implies that the first eigenvalue of the Laplacian will have a lower bound less than $n$ if the Ricci curvature of manifolds involved has a lower bound $n-1$ except on a small part where the Ricci curvature satisfied that $\operatorname{Ric}(M) \geq 0$. Now a natural question arises: what is the lower bound of the first eigenvalue of Laplacian on such a manifold? In [2], Petersen and Sprouse gave a lower bound under the assumption that the bad part of the manifolds is small in the sense of $L^{p}$-norm, where $p$ is a constant larger than half of the dimension of the manifold. In this paper, we are interested in the lower bound of the first eigenvalue under the global pinching of the Ricci curvature and we obtain a universal estimate of this lower bound on a certain class of manifolds.

\section{A Sobolev Constant on the Geodesic Ball}

The Sobolev inequality is one of the most important tools in geometric analysis, and the Sobolev constant plays an important part in the study of this field. In this section, we will obtain a general Sobolev constant only depending on the dimension of the manifold on the geodesic ball with small radius.

Definition 1. Let $B_{p}(R) \subseteq M$ be a geodesic ball with radius $R$; we define the Sobolev constant $C_{s}(R)$ on it to be the infimum among all the constant $C$ such that the inequality $\|f\|_{2 n /(n-2)}^{2} \leq C\|\nabla f\|_{2}^{2}$ holds for all $f \in W_{0}^{1.2}\left(B_{p}(R)\right)$.

Definition 2. Let $B_{p}(R) \subseteq M$ be a geodesic ball with radius $R$; we define the isoperimetric constant $C_{0}(R)$ on it to be the supremum among all the constant $\alpha$ such that the inequality Area $(\partial \Omega) \geq \alpha \operatorname{Vol}(\Omega)^{1-(1 / n)}$ holds for all $\Omega \subseteq B_{p}(R)$ with smooth boundary.

For any fixed point $p$ and radius $R$, Croke proves that the equality $C_{s}(R)=4((n-1) /(n-2))^{2} C_{0}(R)^{-2}$ holds [3], but one expects the constant $C_{s}(R)$ to be independent on the location of the point $p$, under some assumptions. In what follows, we will give an upper bound to $C_{s}(R)$ independent of the point $p$.

Let $M$ be an $n$-dimensional Riemannian manifold, $S M$ is the unit tangent bundle of $M$, and $\pi: S M \rightarrow M$ is the canonical projective map. $\Omega \subseteq M, \xi \in S \Omega, \gamma_{\xi}(t)$ is the 
normalized geodesic from $\pi(\xi)$ with the initial velocity $\xi$. We define some notations as follows:

$$
\tau(\xi)=\sup \left\{\tau>0 \mid \gamma_{\xi}(t) \in \Omega, \forall t \in(0, \tau)\right\} .
$$

$C(\xi)$ is the arc length from $\pi(\xi)$ to the cut locus point along $\gamma_{\xi}(t)$. Consider

$$
\begin{gathered}
U \Omega=\{\xi \in S \Omega \mid C(\xi) \geq \tau(\xi)\} \\
U_{x}=(\pi \mid U \Omega)^{-1}(x) ; \quad \omega_{x}=\frac{\mu_{x}\left(U_{x}\right)}{c_{n-1}} ; \quad \omega=\inf _{x \in \Omega} \omega_{x}
\end{gathered}
$$

where $\mu_{x}$ is the standard surface measure of the unit sphere, $c_{n-1}$ is denoted to be the area of the unit sphere $S^{n-1}$.

Definition 3. Using the Notation above, $\omega=\inf _{x \in \Omega} \omega_{x}$ is called the visibility angle of $\Omega$.

If the manifold has $\operatorname{Inj}(M) \geq i$ which ensures that any minimal geodesic starting from any point in $B_{p}(i / 2)$ will reach the boundary $\partial B_{p}(i / 2)$ before it reaches its cut locus, then the visibility angle of $B_{p}(i / 2)$ for any point $p$ which we denote by $\omega(i / 2)$ satisfies $\omega(i / 2)=1$.

Lemma 4. Let $M^{n}$ be a closed Riemannian manifold with Inj $(M) \geq i$, then for any $p \in M$, the following Sobolev inequality holds on $B_{p}(i / 2):\|f\|_{2 n /(n-2)}^{2} \leq C\|\nabla f\|_{2}^{2}$, where $f \in W_{0}^{1.2}\left(B_{p}(i / 2)\right)$ and $C=C(n)$.

Proof. Croke proved the following inequality [4]:

$$
\frac{\operatorname{Area}(\partial \Omega)}{\operatorname{Vol}(\Omega)^{1-(1 / n)}} \geq \frac{c_{n-1}}{\left(c_{n} / 2\right)^{1-(1 / n)}} \omega^{1+(1 / n)},
$$

where $\Omega \subseteq B_{p}(i / 2), \partial \Omega \in C^{\infty}$, and $\omega$ is just the visibility angle of the domain $\Omega$.

As discussed above, we will have $\omega(\Omega)=1$ if $\Omega \subseteq B_{p}(i / 2)$; then according to Croke's inequality, we obtain $C_{0}(i / 2) \geq$ $c_{n-1} /\left(c_{n} / 2\right)^{1-(1 / n)}$. The relation between $C_{0}(i / 2)$ and $C_{s}(i / 2)$ tells us that $C_{s}(i / 2) \leq C(n)$, where $C(n)$ is a constant only depending on the dimension $n$.

Proposition 5. Let $M^{n}$ be a closed n-dimensional Riemannian manifold with Inj $(M) \geq i$, then for all $p \in M, \operatorname{Vol}\left(B_{p}(i / 2)\right)$ $\geq C_{n} i^{n}$, where $C_{n}$ is a constant only depending on the dimension n.

Proof. Also take the inequality of Croke

$$
\frac{\operatorname{Area}(\partial \Omega)}{\operatorname{Vol}(\Omega)^{1-(1 / n)}} \geq \frac{c_{n-1}}{\left(c_{n} / 2\right)^{1-(1 / n)}} \omega^{1+(1 / n)},
$$

then the result can easily be derived from the fact that $\omega(i / 2)=1$ and Area $\left(\partial B_{p}(r)\right)=d \operatorname{Vol}\left(B_{p}(r)\right) / d r$ after we integrate both sides of the inequality.

\section{The First Eigenfunction and Eigenvalue}

Let $M^{n}$ be a closed $n$-dimensional Riemannian manifold; suppose that $\lambda_{1}(M)$ is the first eigenvalue of the Laplacian and $u$ is the first eigenfunction. In other words, they will satisfy that $\Delta u+\lambda_{1}(M) u=0$. By linearity, we can assume that $-1 \leq u \leq 1$ and $\inf _{x \in M} u=-1$ for the linearity. For the convenience, we call it the normalized eigenfunction. Next we will study some properties of the normalized eigenfunction and the eigenvalue.

Lemma 6. Let $M^{n}$ be a closed $n$-dimensional Riemannian manifold with $\operatorname{Ric}(M) \geq 0$ and $\operatorname{Inj}(M) \geq i$. Then, a constant $C_{1}(n, i)>0$ can be found such that $\lambda_{1}(M) \leq C_{1}(n, i)$.

Proof. One of the theorems of Yau and Schoen [1] shows that $\lambda_{1}(M) \leq E_{n} / d^{2} \leq E_{n} / i^{2} \triangleq C_{1}(n, i)$ if Ric $(M) \geq 0$, where $d$ is the diameter of the manifold and $E_{n}$ is a constant depending only on $n$.

We will now introduce some notation. Let Ric_ $(x)$ denote the lowest eigenvalue of the Ricci curvature tensor at $x$. For a function $f(x)$ on $M^{n}$, we denote $f_{+}(x)=\max \{f(x), 0\}$. Notice that a Riemannian manifold satisfies Ric $\geq n-1$ if and only if $\left((n-1)-\text { Ric }_{-}\right)_{+} \equiv 0$.

The well-known Myers theorem shows that a closed manifold with Ric $\geq n-1$ would have a bounded diameter $d \leq \pi$. In other words, one can deduce that $d \leq \pi$ if one has $(1 / \operatorname{Vol}(M)) \int_{M}\left((n-1)-\mathrm{Ric}_{-}\right)_{+} d \mathrm{vol}=0$. We will show next a result analogous to the one in [5] which we will use in our estimation of the eigenvalue. The proof follows identically; so it will be omitted (the reader can refer to the aforementioned article).

Lemma 7. Let $M^{n}$ be a closed n-dimensional Riemannian manifold with $\operatorname{Ric}(M) \geq 0$, then for any $\delta>0$, there exists $\epsilon_{0}=\epsilon_{0}(n, \delta)>0$ such that if

$$
\frac{1}{\operatorname{Vol}(M)} \int_{M}\left((n-1)-\mathrm{Ric}_{-}\right)_{+} d \mathrm{vol} \leq \epsilon_{0}(n, \delta),
$$

then the diameter will satisfy $d<\pi+\delta$. In particular, there exists $\epsilon_{1}=\epsilon_{1}(n)$ such that if

$$
\frac{1}{\operatorname{Vol}(M)} \int_{M}\left((n-1)-\mathrm{Ric}_{-}\right)_{+} d \operatorname{vol} \leq \epsilon_{1}(n),
$$

then the diameter will satisfy $d<2 \pi$. This fact, together with the volume comparison theorem, implies that $\operatorname{Vol}(M) \leq$ $C_{2}(n)$, where $C_{2}(n)$ is also a constant only dependent of $n$. value.

Now, we can get a rough lower bound for the first eigen-

Lemma 8. For $n \in \mathbb{N}$, let $\epsilon_{1}=\epsilon_{1}(n)>0$ as above and suppose that $M^{n}$ is a closed manifold with

$\operatorname{Ric}(M) \geq 0$,

$$
\frac{1}{\operatorname{Vol}(M)} \int_{M}\left((n-1)-\mathrm{Ric}_{-}\right)_{+} d \mathrm{vol} \leq \epsilon_{1}(n) ;
$$


then there exists a constant $C_{3}(n)>0$ such that $\lambda_{1}(M) \geq$ $\mathrm{C}_{3}(n)$.

Proof. The proof mainly belongs to Li and Yau [6]. Let $u$ be the normalized eigenfunction of $M$, set $v=\log (a+u)$ where $a>1$. Then, we can easily get that

$$
\Delta v=\frac{-\lambda_{1}(M) u}{a+u}-|\nabla v|^{2}
$$

Denote that $Q(x)=|\nabla v|^{2}(x)$, and we then have by the Ricci identity on manifolds with $\operatorname{Ric}(M) \geq 0$ :

$$
\Delta Q=2 v_{i j}^{2}+2 v_{j} v_{j i i} \geq 2 v_{i j}^{2}+2\langle\nabla v, \nabla \Delta v\rangle .
$$

For the term $v_{i j}^{2}$, we have

$$
\sum_{i, j} v_{i j}^{2} \geq \frac{(\Delta v)^{2}}{n} \geq \frac{1}{n}\left(Q^{2}+\frac{2 \lambda_{1}(M) u}{a+u}\right),
$$

and for the term $\langle\nabla v, \nabla \Delta v\rangle$, we have

$$
\langle\nabla v, \nabla \Delta v\rangle=-\frac{a \lambda_{1}(M)}{a+u} Q-\langle\nabla v, \nabla Q\rangle .
$$

Therefore, assume $x_{0} \in M$ to be the maximum of $Q$; then at $x_{0}$, we have

$$
0 \geq \frac{2}{n} Q\left(x_{0}\right)+\left(\frac{4 \lambda_{1}(M)}{n}-\frac{2(n+2) a \lambda_{1}(M)}{n(a-1)}\right) .
$$

Therefore,

$$
Q(x) \leq Q\left(x_{0}\right) \leq \frac{(n+2) a \lambda_{1}(M)}{a-1} .
$$

Denote $\gamma$ to be the minimizing unit speed geodesic joining the maximum and minimum points of $u$; then integrating $Q^{1 / 2}$ along $\gamma$, one will get:

$$
\log \left(\frac{a}{a-1}\right) \leq \log \left(\frac{a+\max u}{a-1}\right) \leq d\left(\frac{(n+2) a \lambda_{1}(M)}{a-1}\right)^{1 / 2} .
$$

Let $t=(a-1) / a$; then for any $t \in\left(\begin{array}{ll}0 & 1\end{array}\right)$, we have $(n+$ 2) $\lambda_{1}(M) \geq t\left(d^{-2}(\log (1 / t))^{2}\right)$.

Considering the maximum of the right hand and the upper bound of the diameter derived in Lemma 7, we can deduce that a positive constant $C_{3}(n)$ can be found such that

$$
\lambda_{1}(M) \geq \frac{4 e^{-2}}{(n+2) d^{2}} \geq C_{3}(n),
$$

where $d$ is the diameter of the manifold.

Corollary 9. If the manifold one discussed satisfies all the conditions in Lemma 8 and its injectivity radius satisfies Inj $(M) \geq i$ and if one let $u$ to be the normalized eigenfunction, then there exists a constant $C_{4}(n, i)>0$ such that $|\nabla u|^{2} \leq$ $C_{4}(n, i)$.
Proof. Set $a=2$ in the (13) from above. Then applying Lemma 6, one obtains

$$
\frac{1}{9}|\nabla u|^{2} \leq|\nabla v|^{2}(x) \leq 2 C_{1}(n, i)(n+2) ;
$$

therefore,

$$
|\nabla u|^{2} \leq C_{4}(n, i)
$$

Proposition 10. Let $M^{n}$ be a closed n-dimensional Riemannian manifold, $u$ the first eigenfunction of the Laplacian, and $\lambda_{1}(M)$ the corresponding eigenvalue, then $\Delta|u|+\lambda_{1}(M)|u| \geq 0$ holds in the sense of distribution. Moreover, if $M^{n}$ is compact with boundary, then the same conclusion holds for its Neumann boundary value problem.

Proof. From the definition, we know that $\Delta u+\lambda_{1}(M) u=0$ holds on $M$. Denote

$$
\begin{aligned}
& M^{+}=\{x \in M \mid u(x)>0\}, \\
& M^{-}=\{x \in M \mid u(x)<0\}, \\
& M^{0}=\{x \in M \mid u(x)=0\} .
\end{aligned}
$$

According to the maximum principle of elliptic equation and the discussion about nodal set and nodal regions in [1], we can conclude that $\partial M^{+}=\partial M^{-}=M^{0}$ is a smooth manifold with dimension $n-1$.

For all $\phi \in C^{\infty}(M), \phi \geq 0$, integrating by parts we then have

$$
\begin{aligned}
\int_{M}|u| \Delta \phi+\lambda_{1}(M) \phi|u| \\
=\int_{M^{+}}|u| \Delta \phi+\lambda_{1}(M) \phi|u|+\int_{M^{-}}|u| \Delta \phi+\lambda_{1}(M) \phi|u| \\
=\int_{\partial M^{+}}\left(u \frac{\partial \phi}{\partial n^{+}}-\phi \frac{\partial u}{\partial n^{+}}\right)+\int_{M^{+}} \phi\left(\Delta u+\lambda_{1}(M) u\right) \\
\quad-\int_{\partial M^{-}}\left(u \frac{\partial \phi}{\partial n^{-}}-\phi \frac{\partial u}{\partial n^{-}}\right)-\int_{M^{-}} \phi\left(\Delta u+\lambda_{1}(M) u\right) \\
=\int_{\partial M^{-}} \phi \frac{\partial u}{\partial n^{-}}-\int_{\partial M^{+}} \phi \frac{\partial u}{\partial n^{+}} \geq 0,
\end{aligned}
$$

where $n^{+}$and $n^{-}$denote the outward normal direction with respect to the boundaries of $M^{+}$and $M^{-}$, respectively. Note that $\partial u / \partial n^{-} \geq 0$ on $\partial M^{-}$and $\partial u / \partial n^{+} \leq 0$ on $\partial M^{+}$for the definition of $\mathrm{M}^{-}$and $\mathrm{M}^{+}$. This completes the proof.

When $M$ has boundary, we can apply the same reasoning, except that the test function will require $\phi \in C_{0}^{\infty}(M)$. This gives the proof.

As long as the given manifold is compact, one knows that the first normalized eigenfunction is then determined. This indicates that the first normalized eigenfunction of the Laplacian has a close relation with the geometry of 
the manifold. In particular, one would hope to bound the $L^{2}$-norm of first normalized eigenvalue of Laplacian from below by the geometric quantities. In this sense, we have the following result.

Theorem 11. Let $M^{n}$ be a closed $n$-dimensional Riemannian manifold with $\operatorname{Ric}(M) \geq 0$ and $\operatorname{Inj}(M) \geq i$. If $u$ is the normalized eigenfunction of the Laplacian, then there exists a constant $C_{5}(n, i)>0$ such that $\int_{M} u^{2} \geq C_{5}(n, i)$.

Proof. We use Moser iteration to get the result. From Proposition 10 , we know that $\Delta|u|+\lambda_{1}(M)|u| \geq 0$ holds on $M$ in the sense of distribution. Set $v=|u|$ and take the point $p \in M$ such that $u(p)=-1$.

For $a \geq 1$, denote $R=i / 2 ; \phi$ is a cut-off function on $B_{p}(R)$, then we have by integrating by parts:

$$
\begin{aligned}
& \lambda_{1}(M) \int_{B_{p}(R)} \phi^{2} v^{2 a}=-\int_{B_{p}(R)} \phi^{2} v^{2 a-1} \Delta v \\
& \quad=2 \int_{B_{p}(R)} \phi v^{2 a-1}+(2 a-1) \int_{B_{p}(R)} \phi^{2} v^{2 a-2}|\nabla v|^{2} \\
& \quad \geq 2 \int_{B_{p}(R)} \phi v^{2 a-1}+a \int_{B_{p}(R)} \phi^{2} v^{2 a-2}|\nabla v|^{2} .
\end{aligned}
$$

However, using the identity

$$
\begin{aligned}
\int_{B_{p}(R)}\left|\nabla\left(\phi v^{a}\right)\right|^{2}= & \int_{B_{p}(R)}|\nabla \phi|^{2} v^{2 a}+2 a \int_{B_{p}(R)} \phi v^{2 a-1}\langle\nabla \phi, \nabla v\rangle \\
& +a^{2} \int_{B_{p}(R)} \phi^{2} v^{2 a-2}|\nabla v|^{2},
\end{aligned}
$$

we have

$$
\lambda_{1}(M) a \int_{B_{p}(R)} v^{2 a} \phi^{2} \geq \int_{B_{p}(R)}\left|\nabla\left(\phi v^{a}\right)\right|^{2}-\int_{B_{p}(R)} v^{2 a}|\nabla \phi|^{2} ;
$$

therefore, using the Sobolev inequality in Lemma 4,

$$
\begin{aligned}
& \lambda_{1}(M) a \int_{B_{p}(R)} v^{2 a} \phi^{2}+\int_{B_{p}(R)} v^{2 a}|\nabla \phi|^{2} \\
& \geq \int_{B_{p}(R)}\left|\nabla\left(\phi v^{a}\right)\right|^{2} \geq \frac{1}{C}\left(\int_{B_{p}(R)}\left(\phi v^{a}\right)^{2 n /(n-2)}\right)^{(n-2) / n} \\
& \quad=\frac{1}{C}\left\|\phi v^{a}\right\|_{2 n /(n-2)}^{2} .
\end{aligned}
$$

Let

$$
\phi= \begin{cases}1, & \text { in } B_{p}(\rho), \\ \frac{\rho+\sigma-r}{\sigma}, & \text { in } \frac{B_{p}(\rho+\sigma)}{B_{p}(\rho)}, \\ 0, & \text { in } \frac{B_{p}(R)}{B_{p}(\rho+\sigma)} .\end{cases}
$$

Putting $\phi$ into the inequality above and we then have by splitting the integral into three parts and using the values of $\phi$ on each of them:

$$
\|v\|_{2 a n /(n-2) ; B_{p}(\rho)} \leq\left[C\left(\lambda_{1}(M) a+\frac{1}{\sigma^{2}}\right)\right]^{1 / 2 a}\|v\|_{2 a ; B_{p}(\rho+\sigma)},
$$

where we denote $\|f\|_{p ; \Omega}=\left(\int_{\Omega} f^{p}\right)^{1 / p}$ only for emphasizing the integral domain.

Set

$$
\begin{aligned}
& \left\{a_{j}\right\}: a_{0}=1, a_{1}=\frac{n}{n-2}, \ldots, a_{j}=\left(\frac{n}{n-2}\right)^{j}, \ldots, \\
& \left\{\sigma_{j}\right\}: \sigma_{0}=\frac{R}{4}, \sigma_{1}=\frac{R}{8}, \ldots, \sigma_{j}=\frac{R}{2^{-(2+j)}}, \ldots, \\
& \left\{\rho_{j}\right\}: \rho_{-1}=R, \ldots, \rho_{j}=R-\sum_{s=0}^{j} \sigma_{l}, \ldots
\end{aligned}
$$

And putting $\left\{a_{j}\right\},\left\{\sigma_{j}\right\},\left\{\rho_{j}\right\}$ into (25), we can derive after iteration that

$$
\begin{aligned}
\|v\|_{2 a_{j+1} ; B_{p}\left(\rho_{j}\right)} & \leq\left[C\left(\lambda_{1}(M) a_{j}+\frac{1}{\sigma_{j}^{2}}\right]^{1 / 2 a_{j}}\|v\|_{2 a_{j} ; B_{p}\left(\rho_{j-1}\right)}\right. \\
& \leq \prod_{s=0}^{j}\left[C\left(\lambda_{1}(M) a_{s}+\frac{1}{\sigma_{s}^{2}}\right)\right]^{1 / 2 a_{s}}\|v\|_{2 ; B_{p}(R)} .
\end{aligned}
$$

Let $j \rightarrow+\infty$, then

$$
\|v\|_{\infty ; B_{p}(R / 2)} \leq \prod_{j=0}^{\infty}\left[C\left(\lambda_{1}(M) a_{j}+\frac{1}{\sigma_{j}^{2}}\right]^{1 / 2 a_{j}}\|v\|_{2 ; B_{p}(R)} .\right.
$$

The product can be estimated as follows:

$$
\begin{aligned}
\prod_{j=0}^{\infty} & {\left[C\left(\lambda_{1}(M) a_{j}+\frac{1}{\sigma_{j}^{2}}\right)\right]^{1 / 2 a_{j}} } \\
& \leq \prod_{j=0}^{\infty}\left[C\left(\lambda_{1}(M)+\frac{16}{R^{2}}\right)\right]^{1 / 2 a_{j}} 4^{j / 2 a_{j}} .
\end{aligned}
$$

The right hand will converge to a fixed number by using the fact that $\prod_{j=0}^{\infty} B^{\mu^{j}}=B^{\mu /(\mu-1)}$ and the fact $\sum_{j=0}^{\infty} j \mu^{-j}$ is finite for some $B \in R, \mu>1$. From $\lambda_{1}(M) \leq C_{1}(n, i)$, we can find a positive constant $C_{5}(n, i)>0$ such that

$$
\|v\|_{\infty ; B_{p}(R / 2)} \leq C_{5}^{-1 / 2}(n, i)\|v\|_{2 ; B_{p}(R)} \leq C_{5}^{-1 / 2}(n, i)\|v\|_{2 ; M} .
$$

Therefore,

$$
\int_{M} u^{2} \geq C_{5}(n, i)
$$




\section{The Lower Bound of the First Eigenvalue}

Using the same notation as above, we can state the following result.

Theorem 12. For $n \in \mathbb{N}, i, \delta \in \mathbb{R}^{+}$, there is an $\epsilon=\epsilon(n, i, \delta)>$ 0 such that any closed manifold $M^{n}$ with $\operatorname{Ric}(M) \geq 0$, $\operatorname{Inj}(M) \geq i$ and

$$
\frac{1}{\operatorname{Vol}(M)} \int_{M}\left((n-1)-\mathrm{Ric}_{-}\right)_{+} d \mathrm{vol} \leq \epsilon
$$

will satisfy that $\lambda_{1}(M) \geq n-\delta$.

Proof. Assume that $u$ is the normalized eigenfunction of Laplacian on $M^{n}$, let $Q(x)=|\nabla u|^{2}+\left(\lambda_{1}(M) / n\right) u^{2}$, direct computation shows that

$$
\begin{aligned}
\frac{1}{2} \Delta Q & =u_{i j}^{2}+u_{j} u_{i i j}+R_{i j} u_{i} u_{j}+\frac{\lambda_{1}(M)}{n}|\nabla u|^{2}+\frac{\lambda_{1}(M)}{n} u \Delta u \\
& \geq \frac{1-n}{n} \lambda_{1}(M)|\nabla u|^{2}+R_{i j} u_{i} u_{j} .
\end{aligned}
$$

Integrating both sides on $M^{n}$, we have

$$
\begin{aligned}
0 \geq & \frac{1-n}{n} \lambda_{1}(M) \int_{M}|\nabla u|^{2} d \mathrm{vol}+\int_{M} R_{i j} u_{i} u_{j} d \mathrm{vol} \\
\geq & \frac{1-n}{n} \lambda_{1}(M) \int_{M}|\nabla u|^{2} d \mathrm{vol}+\int_{M} \mathrm{Ric}_{-}|\nabla u|^{2} d \mathrm{vol} \\
= & \frac{1-n}{n} \lambda_{1}(M) \int_{M}|\nabla u|^{2} d \mathrm{vol}+(n-1) \int_{M}|\nabla u|^{2} d \mathrm{vol} \\
& -\int_{M}\left[(n-1)-\mathrm{Ric}_{-}\right]|\nabla u|^{2} d \mathrm{vol} ;
\end{aligned}
$$

therefore,

$$
\begin{aligned}
\lambda_{1}(M) \geq & n-\frac{\operatorname{Vol}(M)}{\lambda_{1}(M) \int_{M}|u|^{2} d \operatorname{vol}} \frac{1}{\operatorname{Vol}(M)} \\
& \times \int_{M}\left((n-1)-\text { Ric }_{-}\right)_{+}|\nabla u|^{2} d \mathrm{vol}
\end{aligned}
$$

if we suppose that

$$
\frac{1}{\operatorname{Vol}(M)} \int_{M}\left((n-1)-\mathrm{Ric}_{-}\right)_{+} d \mathrm{vol} \leq \epsilon_{1}(n) .
$$

If $\epsilon_{1}$ is the one obtained in Lemma 7, then one has:

$$
\lambda_{1}(M) \geq n-\frac{C_{2} C_{4}}{C_{3} C_{5}} \frac{1}{\operatorname{Vol}(M)} \int_{M}\left((n-1)-\text { Ric }_{-}\right)_{+} d \text { vol. }
$$

Finally, if one chooses

$$
0<\epsilon=\epsilon(n, i, \delta) \leq \min \left\{\epsilon_{1}, \delta \frac{C_{3} C_{5}}{C_{2} C_{4}}\right\}
$$

then $\lambda_{1}(M) \geq n-\delta$ as long as $(1 / \operatorname{Vol}(M)) \int_{M}((n-1)-$ $\left.\mathrm{Ric}_{-}\right)_{+} d \mathrm{vol} \leq \epsilon$, and this proves the theorem.

\section{Acknowledgments}

The authors owe great thanks to the referees for their careful efforts to make the paper clearer. Research of the first author was supported by STPF of University (no. J11LA05), NSFC (no. ZR2012AM010), the Postdoctoral Fund (no. 201203030) of Shandong Province, and Postdoctoral Fund (no. 2012M521302) of China. Part of this work was done while the first author was staying at his postdoctoral mobile research station of QFNU.

\section{References}

[1] S. T. Yau and R. Schoen, Lectures in Differential Geometry, Scientific Press, 1988.

[2] P. Petersen and C. Sprouse, "Integral curvature bounds, distance estimates and applications," Journal of Differential Geometry, vol. 50, no. 2, pp. 269-298, 1998.

[3] D. Yang, "Convergence of Riemannian manifolds with integral bounds on curvature. I," Annales Scientifiques de l'École Normale Supérieure. Quatrième Série, vol. 25, no. 1, pp. 77-105, 1992.

[4] I. Chavel, Riemannian Geometry: A Mordern Introduction, Cambridge University Press, Cambridge, UK, 2000.

[5] C. Sprouse, "Integral curvature bounds and bounded diameter," Communications in Analysis and Geometry, vol. 8, no. 3, pp. 531$543,2000$.

[6] P. Li and S. T. Yau, "Estimates of eigenvalues of a compact Riemannian manifold," in AMS Proceedings of Symposia in Pure Mathematics, pp. 205-239, American Mathematical Society, Providence, RI, USA, 1980. 


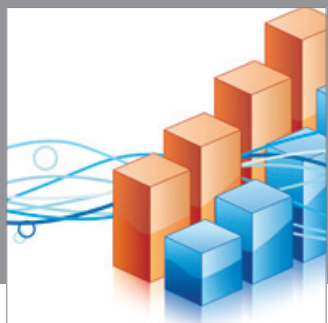

Advances in

Operations Research

mansans

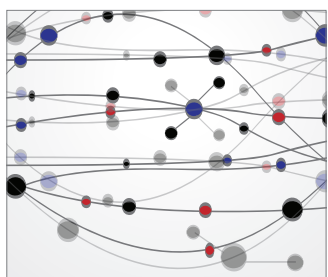

The Scientific World Journal
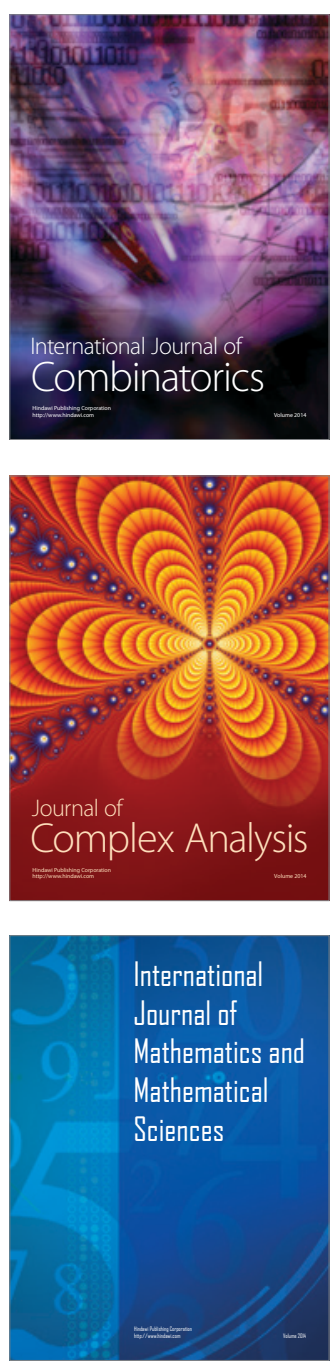
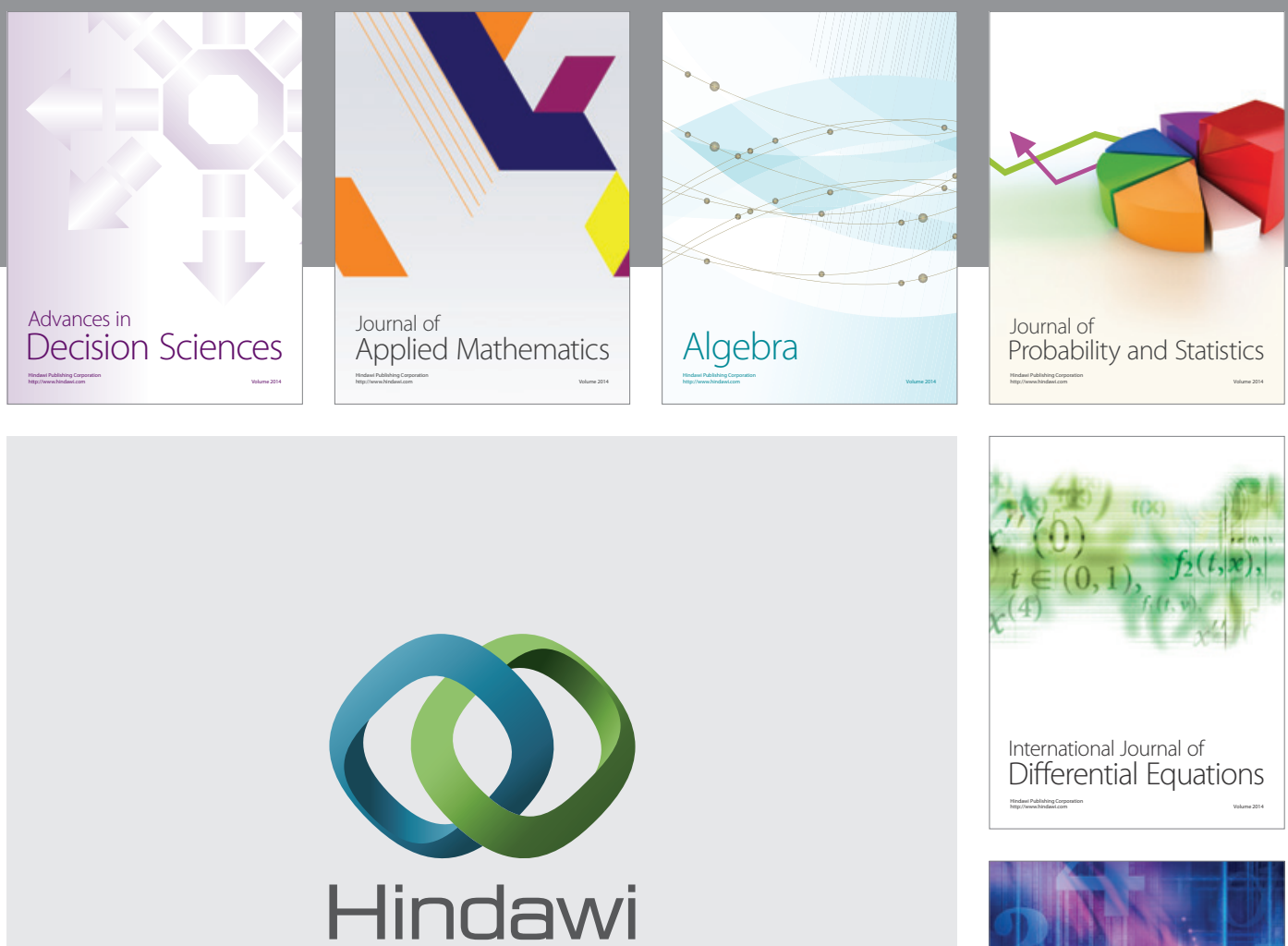

Submit your manuscripts at http://www.hindawi.com
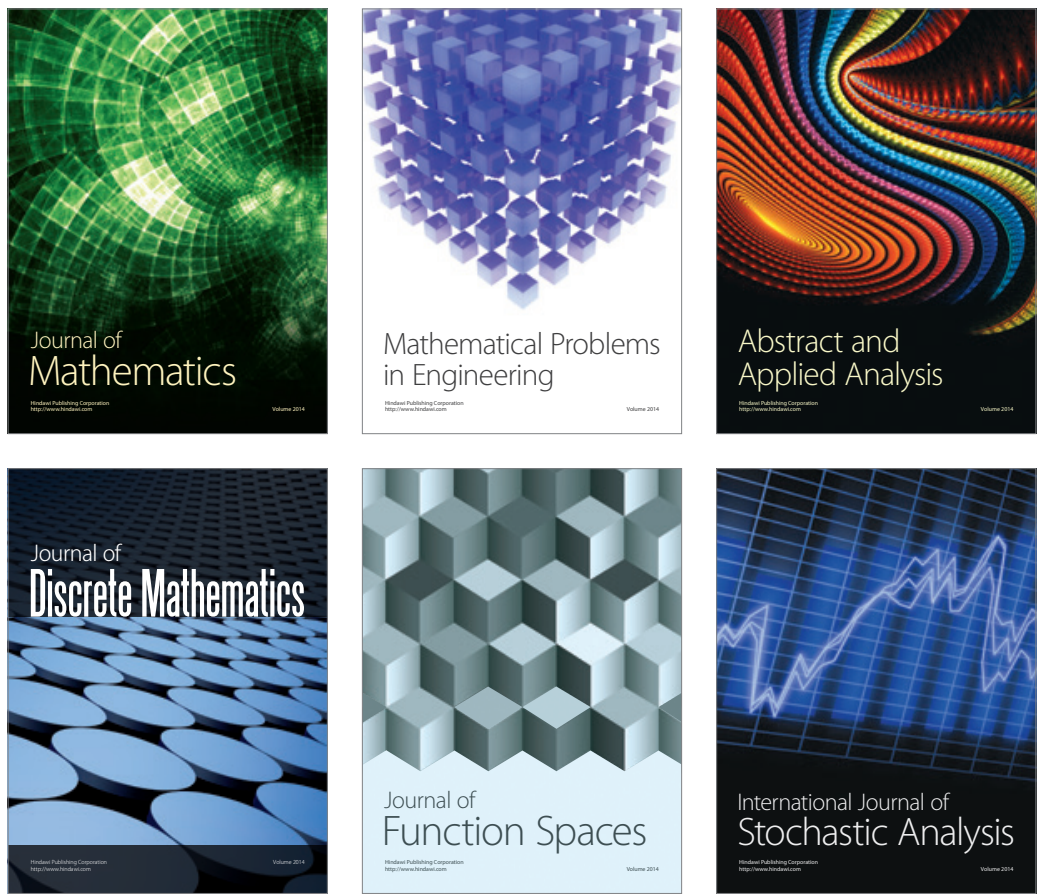

Journal of

Function Spaces

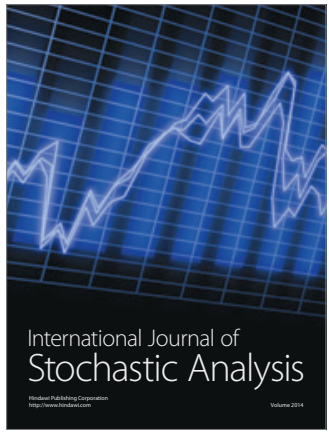

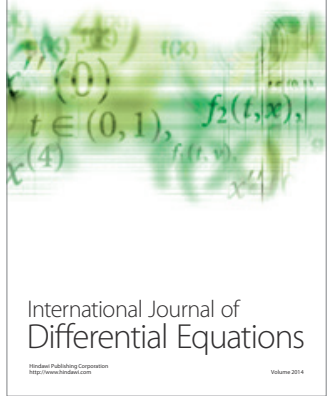
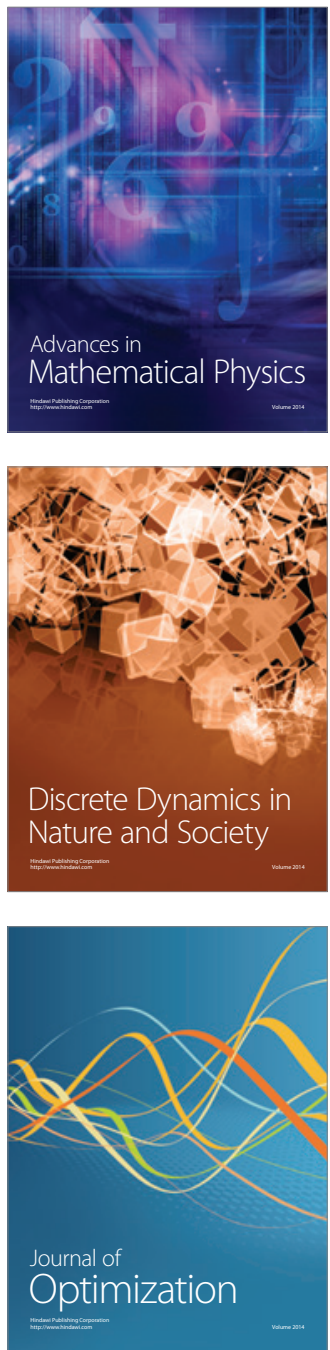